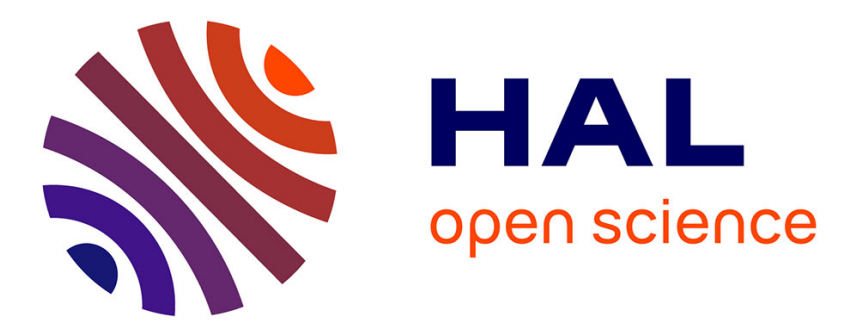

\title{
Theoretical study of electronic excitation, ion-pair formation, and mutual neutralization in cesium-hydrogen collisions
}

\author{
Andrey Belyaev, Bruno Lepetit, Florent X. Gadéa
}

\section{To cite this version:}

Andrey Belyaev, Bruno Lepetit, Florent X. Gadéa. Theoretical study of electronic excitation, ionpair formation, and mutual neutralization in cesium-hydrogen collisions. Physical Review A : Atomic, molecular, and optical physics [1990-2015], 2014, 90, pp.062701. 10.1103/PhysRevA.90.062701 . hal01089958

\section{HAL Id: hal-01089958 https://hal.science/hal-01089958}

Submitted on 3 Dec 2014

HAL is a multi-disciplinary open access archive for the deposit and dissemination of scientific research documents, whether they are published or not. The documents may come from teaching and research institutions in France or abroad, or from public or private research centers.
L'archive ouverte pluridisciplinaire HAL, est destinée au dépôt et à la diffusion de documents scientifiques de niveau recherche, publiés ou non, émanant des établissements d'enseignement et de recherche français ou étrangers, des laboratoires publics ou privés. 


\title{
Theoretical study of electronic excitation, ion-pair formation, and mutual neutralization in cesium-hydrogen collisions
}

\author{
Andrey K. Belyaev* \\ Department of Theoretical Physics, Herzen University, St. Petersburg 191186, Russia \\ Bruno Lepetit \\ Université de Toulouse, UPS, Laboratoire Collisions Agrégats Réactivité, IRSAMC, F-31062 Toulouse, France and CNRS, UMR 5589, \\ F-31062 Toulouse, France \\ Florent Xavier Gadéa \\ Université de Toulouse, UPS, Laboratoire de Chimie et Physique Quantiques, IRSAMC, F-31062 Toulouse, France and CNRS, UMR 5626, \\ F-31062 Toulouse, France \\ (Received 17 October 2014; published 1 December 2014)
}

\begin{abstract}
Inelastic cross sections for the excitation, deexcitation, ion-pair formation, and mutual neutralization processes in cesium-hydrogen collisions $\mathrm{Cs}(6 s, 6 p, 5 d, 7 s)+\mathrm{H}$ and $\mathrm{Cs}^{+}+\mathrm{H}^{-}$are calculated by means of the recently proposed branching-probability-current method and the recently calculated accurate $a b$ initio adiabatic potential energies. Scattering calculations are performed in the low-energy range from $0.01 \mathrm{eV}$ to $1 \mathrm{keV}$. It is shown that among the endothermic processes, the highest values of the partial cross sections correspond to the ion-pair formation processes with the maximum values up to $23 \AA^{2}$. Among the exothermic processes in the low-energy range, the largest partial cross section corresponds to the mutual neutralization process into the $\mathrm{Cs}(5 d)+\mathrm{H}$ final state.
\end{abstract}

DOI: 10.1103/PhysRevA.90.062701

\section{INTRODUCTION}

Negative-ion sources are used as a major heating system in nuclear fusion reactors. They involve the formation of a hydrogen-negative-ion $\left(\mathrm{H}^{-}\right.$or $\left.\mathrm{D}^{-}\right)$beam subsequently accelerated, neutralized, and injected in the fusion plasma. The negative ions are thought to be produced by volume or surface processes, as described in [1-4].

Volume production involves dissociative attachment of lowenergy electrons to vibrationally excited molecular hydrogen: $\mathrm{H}_{2}(v \geqslant 5)+e(\leqslant 1 \mathrm{eV}) \rightarrow \mathrm{H}^{-}+\mathrm{H}$. Several processes can be invoked to produce vibrationally excited hydrogen. Molecular hydrogen can be vibrationally or electronically excited by collisions with high-energy electron impact, followed by radiative decay in the latter case. Vibrationally excited molecular hydrogen can also be produced by atomic hydrogen recombination near surfaces $[5,6]$. The negative-ion main destruction processes are mutual neutralization with positive ions, electron detachment, and associative detachment in collisions with neutral hydrogen. As the mean free paths associated with these processes are typically of the order of several centimeters [7], negative ions should be produced within this distance from the extraction region in order to be present in the ion beam.

In addition to volume production, surface production can be invoked to explain the formation of negative hydrogen ions. In this case, neutral or positive ions impacting a surface capture electrons in their affinity levels. This process requires a minimum collision energy given by the difference between the surface electron work function and the affinity level energy [8].

\footnotetext{
*Corresponding author: andrey.k.belyaev@gmail.com
}

PACS number(s): 34.10.+x, 34.50.Fa, 34.70.+e

High-intensity negative-ion currents are necessary to achieve fusion plasma heating requirements. Such negativeion current density enhancement by typically one order of magnitude can be achieved by introduction of cesium into the source $[8,9]$. This enhancement is usually explained by a more efficient surface production mechanism (see, for instance, $[10,11])$. Indeed, covering a surface with cesium is expected to lower its work function, decrease the electroncapture threshold, and increase the negative-ion production rate. Other processes may also produce negative ions from low work function surfaces, for instance, the $\mathrm{H}_{2}{ }^{-}$molecular ion formed at the surface from the neutral molecule (in turn formed from $\mathrm{H}_{3}{ }^{+}$or $\mathrm{H}_{2}{ }^{+}$neutralization at the surface) and subsequently dissociating [12].

However, there is recent experimental evidence that surface production is not the only contributor to negative-ion production enhancement in cesium seeded sources. Indeed, the surface production mechanism can be inhibited [8] by setting the producing surface to a high positive potential, confining the negative ions in its vicinity. Under such conditions, the measured negative-ion current has to originate from a volume production mechanism. This contribution exists even without cesium as described above; it is large and is enhanced by a factor of the order of 3 when cesium is present in the chamber $[8,9]$. Several processes can be invoked to explain this enhanced negative-ion volume production [4]. One possibility could be that the Cs enhancement effect is indirect and mediated by larger production of vibrationally excited $\mathrm{H}_{2}$, for instance, through $\mathrm{Cs}+\mathrm{H}_{3}{ }^{+}$collisions [13] or electronic to vibrational energy conversion in $\mathrm{Cs}^{*}+\mathrm{H}_{2}$ inelastic collisions [14]. However, it was recently shown that molecular hydrogen VUV emission intensity was not affected by the presence or absence of Cs [15]. As this VUV emission is related to the formation of $\mathrm{H}_{2}$ vibrationally excited states from electronically excited ones, it was concluded that the presence 
of cesium has little effect on the formation of vibrationally excited $\mathrm{H}_{2}$ and thus on the subsequent production of $\mathrm{H}^{-}$ through dissociative attachment.

It is therefore likely that other $\mathrm{H}^{-}$volume production mechanisms sensitive to the presence of cesium take place. One possibility is dissociative attachment to $\mathrm{CsH}$ molecules [16]. $\mathrm{CsH}$ molecules may be formed from the $\mathrm{Cs}+\mathrm{H}_{2}$ reaction (which is endothermic by less than $3 \mathrm{eV}$ when reactants and products are in their ground states). This reactive process is efficient when Cs is electronically excited or when $\mathrm{H}_{2}$ is vibrationally excited [14]. However, little is currently known about the dissociative attachment process. Another possible $\mathrm{H}^{-}$volume formation process is the direct collisional ion-pair formation $\mathrm{Cs}+\mathrm{H} \rightarrow \mathrm{Cs}^{+}+\mathrm{H}^{-}$. This process simultaneously provides ionized cesium and it is known from emission line intensity measurements that ionized species have densities several orders of magnitude larger than neutral ones [17]. This suggests ion-pair formation as a candidate for hydrogen negative-ion production. We now focus on this process and its inverse, mutual neutralization.

Results on this process are reviewed in [18]. Experiments were restricted to energies above $100 \mathrm{eV}$ (see [19,20] and references therein), which are not relevant in the present context. Most calculations [21-25] were also performed similarly at high collision energy, using integration of coupled equations along a classical trajectory assumed to be a straight line. Some calculations [23-25] provided good agreement with experimental results $[19,20]$ above $100 \mathrm{eV}$ for ion-pair formation from $\operatorname{Cs}(6 s)$. For the low energies of interest here, the only available results are the calculations from Ref. [26]. They provided cross sections for the four $6 s, 6 p, 7 s$, and $7 p$ states with the Landau-Zener theory in the energy range $0.01-10000 \mathrm{eV}$. The fact that these results deviate significantly from the other calculations as well as from the experimental results in the high-energy range (Fig. 45 in [18]) justifies the need for a new calculation of these processes. This is the aim of the present paper, to present new mutual neutralization as well as an ion-pair formation cross section for the $\mathrm{CsH}$ system using recent $a b$ initio potentials. Section II presents these potentials, Sec. III the method used for the dynamical calculation, and Sec. IV the calculated cross sections, which are discussed with respect to available data.

\section{ELECTRONIC POTENTIALS}

Inelastic processes in cesium-hydrogen collisions are treated within the Born-Oppenheimer formalism, which is the most widely used and reliable approach for theoretical studies of heavy-particle collisions. The first step in this formalism is determination of the electronic structure of a treated molecule.

We use in the present study the recent adiabatic potentials from Ref. [27], which improve previous results [28,29]. A full valence configuration-interaction calculation has been performed reducing the cesium atom to a single-electron atom with a pseudopotential. A core polarization potential is used to describe the interaction between the $\mathrm{Cs}^{+}$polarizable core with valence electrons and $\mathrm{H}$ nucleus. Gaussian-type orbitals including diffuse ones are used to describe the valence electrons. The resulting potentials are shown in Fig. 1. They present undulations that are related to the intrinsic

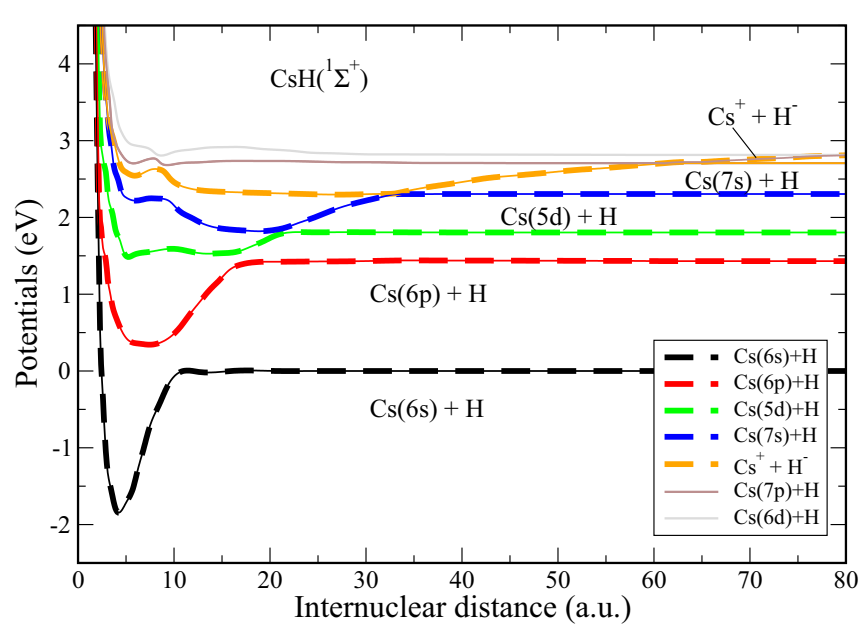

FIG. 1. (Color online) Adiabatic potential curves (thin solid lines) for the low-lying $\mathrm{CsH}\left({ }^{1} \Sigma^{+}\right)$states up to and including the ionic one. The thick dashed lines indicate the potentials that are used in the present nonadiabatic nuclear dynamical calculations: The four lowest potentials coincide with the corresponding adiabatic potentials; the fifth potential coincides with the fifth lowest adiabatic potential at the internuclear distance $R<61$ a.u. and at $R>61$ a.u. it is diabatically extended to the Coulomb potential of the ionic $\mathrm{Cs}^{+}+\mathrm{H}^{-}$state.

characteristics of the Rydberg states that extend to large internuclear distances.

The dominant qualitative feature is a neutral-ionic interaction that induces avoided crossings between an ionic curve that asymptotically correlates to the ion-pair state $\mathrm{Cs}^{+}+\mathrm{H}^{-}$and shows a Coulomb shape and neutral potentials that correlate to the neutral $\operatorname{Cs}(6 s, 6 p, 5 d, 7 s)+\mathrm{H}$ states. This interaction generates single deep wells in the two lowest potentials, the ground $X$ and first excited $A^{1} \Sigma^{+}$states. More excited states can have multiple-well structures. The asymptotic excitation energies are $1.43 \mathrm{eV}(6 p), 1.80 \mathrm{eV}(5 d)$, and $2.30 \mathrm{eV}(7 s)$. The agreement between these and the experimental values is better than $0.01 \mathrm{eV}$. The avoided-crossing distance increases as higher excited states are considered. Simultaneously, it is clear in Fig. 1 that the interacting states avoid each other less and less, which means that the strength of their interaction decreases. Based on the previous experience with quantum [30-33] and model [34] scattering calculations, we have therefore not included in our calculation $\operatorname{Cs}(7 p)$ and more excited covalent states; they are not expected to contribute to ion-pair formation or mutual neutralization.

\section{NONADIABATIC NUCLEAR DYNAMICAL METHOD}

The second step of the Born-Oppenheimer formalism is a nonadiabatic nuclear dynamical treatment. Known adiabatic potentials [27] allow one to estimate inelastic cross sections and rate coefficients by means of the model approach [34] based on the branching-probability-current method for the nonadiabatic nuclear dynamics. The branching-probabilitycurrent method is an extension of the branching-classicaltrajectory method developed in Ref. [35]. Both methods assume that a nonadiabatic transition in each nonadiabatic avoided-crossing region can be described within the LandauZener (LZ) model. In this case, a nonadiabatic transition 
probability $p_{j k}$ after a single traverse of a nonadiabatic region can be evaluated by means of the LZ formula

$$
p_{j k}^{\mathrm{LZ}}=\exp \left(-\xi_{j k} / v\right)
$$

where $\xi_{j k}$ is a LZ parameter associated with the avoided crossing between states $j$ and $k$ and $v$ is a radial velocity of colliding atoms. All values are evaluated at the center of the corresponding nonadiabatic region. The positions of the nonadiabatic regions $R_{c}$ are determined by local minima of adiabatic splittings $Z_{j k}(R)=\left|U_{j}(R)-U_{k}(R)\right|$ between adjacent adiabatic states $(k=j \pm 1), U_{j}(R)$ and $U_{k}(R)$ being adiabatic potentials. Several nonadiabatic regions between low-lying $\mathrm{CsH}$ molecular states are found in this way based on the $a b$ initio adiabatic potentials at relatively short internuclear distances, as well as a series of avoided crossings at longrange distances due to the ionic-covalent interaction. In each nonadiabatic region the LZ parameters can be calculated by the so-called adiabatic-potential-based formula [34,35]

$$
\xi_{j k}=\left.\frac{\pi}{2 \hbar} \sqrt{\frac{Z_{j k}^{3}}{Z_{j k}^{\prime \prime}}}\right|_{R=R_{c}} .
$$

Primed quantities are referred to as derivatives with respect to the internuclear distance $R$. Equation (2) expresses the LZ parameter only in terms of the adiabatic splitting $Z_{j k}$ and its second distance derivative at $R_{c}$.

It should be emphasized that the conventional LZ formula expresses a LZ parameter in terms of a two-state diabatic representation, which requires a nonuniquely defined diabatization procedure if only adiabatic potentials are known. If an electronic structure is known in a multistate diabatic representation, as in the present case [27], an additional transformation should be performed since two-state matrix elements deviate from multistate ones (see, e.g., Ref. [32]) and the $\mathrm{LZ}$ model requires information about the two-state matrix elements. The advantage of Eq. (2) is that no diabatization procedure is required for calculating $\mathrm{LZ}$ parameters and hence LZ transition probabilities.

Having the possibility to calculate nonadiabatic transition probabilities $p_{j k}$ in each nonadiabatic region, one can calculate an inelastic probability $P_{i f}$ for the transition $i \rightarrow f$, taking into account many nonadiabatic transitions $j \rightarrow k$ occurring between the considered molecular states in any order in which the transitions appear during a collision by means of the branching-probability-current method [34]. The branchingprobability-current method treats a collision as an evolution of probability currents in molecular states along the internuclear distance starting in the asymptotic region, going downward to classical turning points, and then going out to the asymptotic region. After each traverse of a nonadiabatic region, each probability current branches into two currents, keeping the old path along the old effective adiabatic-potential-energy curve (PEC) and creating a new path along a new adiabatic PEC coupled in this nonadiabatic region with the old state; each of these currents is determined by the old current and the nonadiabatic transition probability $p_{j k}$ in this nonadiabatic region. This approach is similar to the splitting of quantum probability currents taking place in rigorous quantum treatments of inelastic atomic collisions. Thus, the code starts with a single incoming current and then generates new currents after traversing nonadiabatic regions according to all possible pathways. A final transition probability $P_{i f}$ is calculated as a sum of all outgoing probability currents for a final state $f$ in the asymptotic region $(R \rightarrow \infty)$ over all pathways created from an initial-channel incoming probability current by branching currents into all nonadiabatic regions in any order that they appear during a collision. No classical trajectory is calculated.

Thus, the branching-probability-current method is twofold: (i) branching a probability current after a traverse of a nonadiabatic region and (ii) the adiabatic-potential-based formula (2) for LZ nonadiabatic transition probabilities. The method has been tested on ion-pair formation processes in $\mathrm{Na}+\mathrm{H}$ collisions [34], showing good agreement with a full quantum calculation [32]. The recent study [36] of mutual neutralization in $\mathrm{H}^{-}+\mathrm{H}^{+}$collisions by means of the $\mathrm{LZ}$ model also shows that the LZ model with proper LZ parameters can yield agreement with a full quantum calculation [37]. This provides additional confidence in the correctness of applying the LZ model, which is the main assumption of the branching-probability-current method. In the present work, the LZ parameters are calculated from the accurate $a b$ initio electronic-structure calculations [27] and thus yield reliable cross sections. The branching-probability-current method has been applied to aluminium-hydrogen [34,38] and to lithiumhelium ion-atom [39] inelastic collisions.

Inelastic cross sections are calculated from inelastic transition probabilities as a sum over partial waves (see, e.g., [34]). Statistical weight factors for initial channels are included in all inelastic cross-section calculations.

\section{RESULTS}

The inelastic cross sections calculated in the present work for collisions $\mathrm{Cs}(6 s, 6 p, 5 d, 7 s)+\mathrm{H}$ and $\mathrm{Cs}^{+}+\mathrm{H}^{-}$are presented in Figs. 2-6 for the collision energy range from the energy thresholds to $1 \mathrm{keV}$. Figure 2 shows the partial and the total cross sections for different final states in $\mathrm{Cs}(6 s)+\mathrm{H}$ collisions when both atoms are initially in their ground states. It is seen that at low collision energies (roughly for the energies below $30 \mathrm{eV}$ ) the largest cross section corresponds to excitation into the first excited state $\operatorname{Cs}(6 p)$. The corresponding mechanism is based on the nonadiabatic (avoided-crossing) region between the ground $X$ and the first excited $A^{1} \Sigma^{+}$ molecular states in the vicinity of the internuclear distance $R \approx 10$ a.u. When the ionic channel is closed, the second largest cross sections correspond to $\mathrm{Cs}(5 d)$ and then to $\mathrm{Cs}(7 s)$ excitation. When the ionic channel is open, the ion-pair formation cross section exceeds that of $\operatorname{Cs}(5 d, 7 s)$ and at the collision energy $E>30 \mathrm{eV}$ it has the highest value, since the molecule traverses the high-lying nonadiabatic regions diabatically. Thus, in the high-energy regime, the ion-pair formation process can be approximately described by the single nonadiabatic region around $R \approx 10$ a.u. with nearly diabatic passing through higher-lying regions. In the lowenergy regime, multiple nonadiabatic regions are involved in the nonadiabatic nuclear dynamics distributing probability currents between different molecular channels (see below) and this should be taken into account for reliable low-energy scattering calculations of inelastic cross sections. 


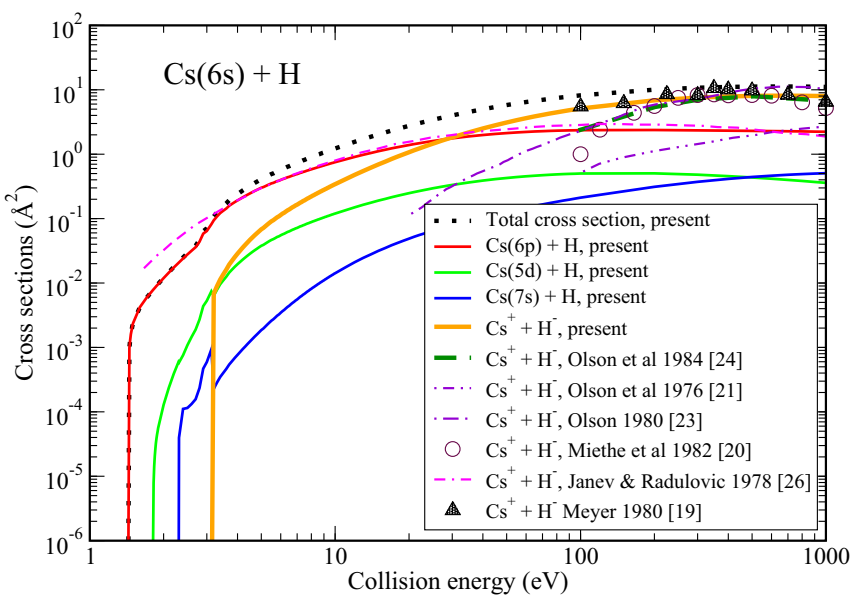

FIG. 2. (Color online) Electronic-excitation and ion-pair formation cross sections as a function of collision energy. For all transitions shown, the initial state is the ground one $\mathrm{Cs}(6 s)+\mathrm{H}$ and the final-state labels are given in the legend. The neutral $\mathrm{Cs}(6 p, 5 d, 7 s)+\mathrm{H}$ and the ionic $\mathrm{Cs}^{+}+\mathrm{H}^{-}$final states are considered. Total cross section refers to the sum over all final states considered in the figure. For the final ionic state (ion-pair formation), the present result is compared with those of Meyer [19], Miethe et al. [20], Olson et al. [21,23,24], and Janev and Radulović [26]. The data of [21] are reduced by a statistical weight factor of $1 / 4$ for the initial channel; the factor was not included in that paper.

Figure 2 compares the present result for the ion-pair formation process in $\mathrm{Cs}(6 s)+\mathrm{H}$ collisions with available experimental data [19,20] and previous calculations [21,23,24,26]. It should be emphasized that the previous studies of inelastic cesium-hydrogen collisions, except for [26], were performed for high energies, typically for $E>1 \mathrm{keV}$ (see Refs. [18,24,25] for references and discussion). In the highenergy range, different experimental and theoretical data, except for [26], agree reasonably well with each other (see Refs. $[18,24,25])$, but at lower energies the disagreement is greater. For example, the only available experimental data for $E<1 \mathrm{keV}[19,20]$ deviate from each other by a factor of 5.5 at $E=100 \mathrm{eV}$ (see Fig. 2). The present calculation for the ion-pair formation process from the $\mathrm{Cs}(6 s)$ state is in good agreement with the experimental data of Ref. [19] in the whole region where these experimental data are available, as well as with other experimental and theoretical data in the high-energy range, except for the data of Ref. [26]. The data of [26] are much lower than all other high-energy theoretical calculations and experimental measurements and cannot be considered as reliable data, although so far they are the only computation available at low energies. It is written in Ref. [26] that their "method for calculating the reaction cross sections, however, is not so accurate." Notice the data from Ref. [26] in Fig. 2 of the present paper are taken from Fig. 5 for ion-pair formation in collisions with $\mathrm{D}$ and are not consistent with the data for the inverse process, mutual neutralization, shown in Fig. 2 of that paper (see also Fig. 6 of the present paper).

Among theoretical calculations the data of Ref. [24] reproduced later in Ref. [25] could be assumed to be the most accurate (having a confidence level of $\pm 50 \%$ according to the authors' estimates). They are based on molecular structure calculations and the close-coupled perturbed-stationary-state calculations with the electron translation factors for a pseudotwo-electron system and classical trajectories for the heavyparticle motion. On the other hand, it was pointed out in Ref. [24] that for scattering calculations the positions and potential-energy differences at the avoided crossings between the ion-pair and covalent states are of importance. The adiabatic potentials were calculated in Ref. [24] by using 14 Slater-type-orbital basis sets. The electronic-structure calculations used in the present work are based on a full valence configuration-interaction calculation [27] and are more accurate than those from Ref. [24]. The adiabaticpotential differences (splittings) $Z_{12}\left(R_{c}\right)$ between the ground $X^{1} \Sigma^{+}$and the first excited $A^{1} \Sigma^{+}$molecular states at the avoided-crossing region at $R \approx 10$ a.u. are $0.656 \mathrm{eV}$ [24] and $0.558 \mathrm{eV}$ [27], respectively. Thus, it results in the $0.1-\mathrm{eV}$ deviation in the adiabatic splittings and this substantially affects the nonadiabatic nuclear dynamics [see, e.g., Eq. (2)]. Of course, the splitting of $0.656 \mathrm{eV}$ [24] is more accurate than the corresponding splittings of $0.82 \mathrm{eV}$ [21] and $0.694 \mathrm{eV}$ [23] calculated in other papers, but the deviation of $0.1 \mathrm{eV}$ (and even more) affects drastically nonadiabatic transition probabilities and inelastic cross sections for the ion-pair formation process at low collision energies. The example is the ion-pair formation cross section calculated in Ref. [23], which decreases too quickly with decreasing collision energy down to $20 \mathrm{eV}$ (see Fig. 2). This steep decrease in the low-energy range is the result of the too large adiabatic splitting at the corresponding nonadiabatic region; at low energies, nonadiabatic transition probabilities and cross sections are very sensitive to a value of a large adiabatic splitting [see Eqs. (1) and (2) as well as Refs. [40,41]]. Similar results hold for other nonadiabatic regions: The adiabatic splittings in nonadiabatic regions calculated in Ref. [24] are smaller than those computed in Ref. [27].

The relevant adiabatic splittings $Z_{j k}$ were obtained in Ref. [26] by means of the semiempirical asymptotic method followed by the two-channel Landau-Zener estimates of nonadiabatic transition probabilities with some corrections for the depletion of the flux to excited states. The comparison of the splittings obtained in Refs. [26,27] shows that the splittings obtained in Ref. [26] are overestimated, e.g., in Ref. [26] the splitting at $R \approx 10$ a.u. is estimated by $0.602 \mathrm{eV}$, and this affects nonadiabatic transition probabilities. In addition, using the two-channel approximation, even corrected by the results of Ref. [42], leads to marked uncertainties in the nonadiabatic nuclear dynamics, especially at low energies. Thus, the analysis of the previous experimental and theoretical data shows that there were no reliable data for low-energy inelastic cesium-hydrogen collisions, although these data are needed for low-temperature plasma studies. The present work provides these data based on accurate $a b$ initio calculations of the adiabatic potentials and on the model nonadiabatic nuclear dynamical treatment. Though quantum close-coupled dynamical calculations are expected to be more accurate than model ones, the present calculations use the most accurate ab initio adiabatic potentials [27] and the physically reasonable model approach [34], both making the present data reliable at low collision energies.

Figure 3 shows the excitation, deexcitation, and ion-pair formation cross sections for $\mathrm{Cs}(6 p)+\mathrm{H}$ collisions. It is 


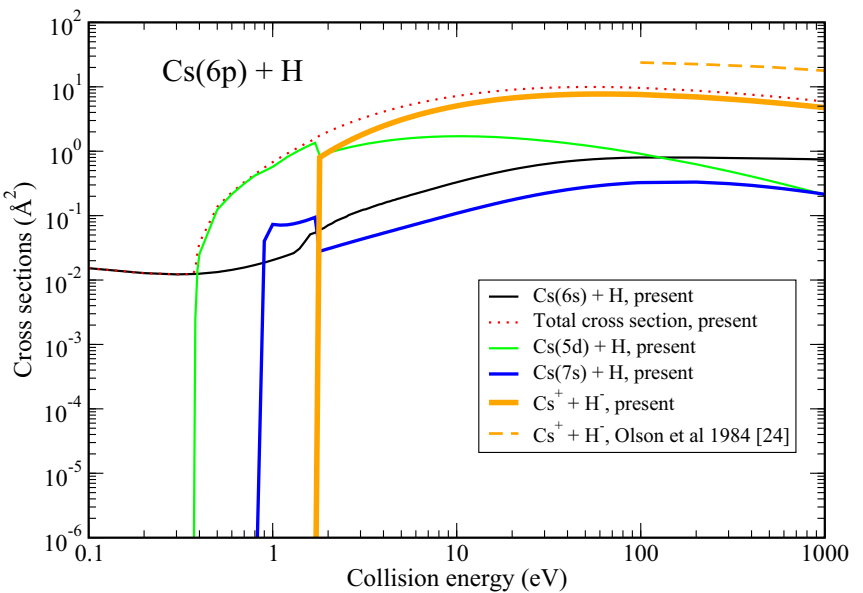

FIG. 3. (Color online) Electronic-excitation cross sections as a function of collision energy. For all transitions shown, the initial state is $\mathrm{Cs}(6 p)+\mathrm{H}$ and the final-state labels are given in the legend. Both neutral $\mathrm{Cs}(6 s, 5 d, 7 s)+H$ and ionic $\mathrm{Cs}^{+}+\mathrm{H}^{-}$final states are considered. Total cross section refers to the sum over all final states considered in this figure. For the final ionic state, the present result is compared with those of Olson et al. [24].

seen that when the ionic channel is closed, the dominant process in these collisions is excitation of the nearest state $\mathrm{Cs}(5 d)$. In this case the probability currents are distributed between different molecular states and oscillate in different adiabatic-potential wells. As soon as the ionic channel is open, the oscillations disappear, the outgoing probability current predominantly populates the ionic channel, the dominant process corresponds to ion-pair formation, and cross sections for populating lower-lying covalent states steeply decrease. The same figure presents a comparison with the data from Ref. [24] for the ion-pair formation process at high collision energies. Again, the disagreement comes from the different electronic-structure calculations used in the present work and in Ref. [24].

Situations similar to the one in $\operatorname{Cs}(6 p)+\mathrm{H}$ collisions are observed in $\mathrm{Cs}(5 d)+\mathrm{H}$ and $\mathrm{Cs}(7 s)+\mathrm{H}$ collisions (Figs. 4 and 5, respectively). At collision energies higher than the energy thresholds for ion-pair formation processes, the ionpair formation cross sections have the highest values. The dominant reaction mechanisms are determined by avoidedcrossing regions due to the ion-covalent interactions, although many nonadiabatic regions are involved into the nonadiabatic nuclear dynamics, resulting in a population of several final channels for each given initial channel. The maximum values for endothermic processes typically do not exceed $10 \AA^{2}$ for the ion-pair formation processes, although the ion-pair formation cross section in $\operatorname{Cs}(7 s)+\mathrm{H}$ collisions can reach the value of $23 \AA^{2}$ in the energy-threshold region (see Figs. 2-5).

Of special interest are the mutual neutralization processes. The corresponding cross sections are collected in Fig. 6. In the low-energy range, the dominant process is mutual neutralization into the $\mathrm{Cs}(5 d)+\mathrm{H}$ final state. This partial mutual neutralization process is predominantly determined by the avoided-crossing nonadiabatic regions at $R \approx 21$ a.u., which lies in the optimal window as discussed in Ref. [34]. This final channel was not treated in Ref. [26], which is the only

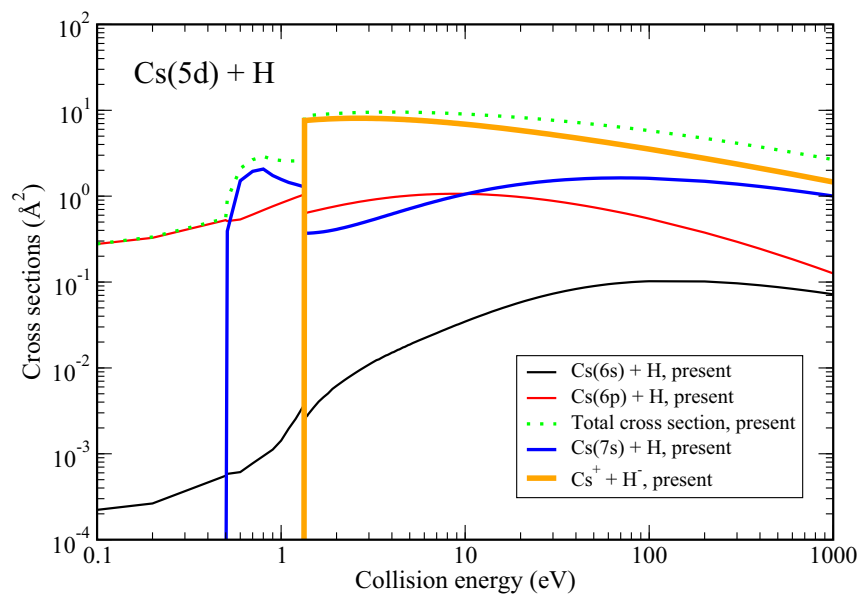

FIG. 4. (Color online) Electronic-excitation cross sections as a function of collision energy. For all transitions shown, the initial state is $\mathrm{Cs}(5 d)+\mathrm{H}$ and the final-state labels are given in the legend. Both neutral $\mathrm{Cs}(6 s, 6 p, 7 s)+\mathrm{H}$ and ionic $\mathrm{Cs}^{+}+\mathrm{H}^{-}$final states are considered. Total cross section refers to the sum over all final states considered in this figure.

previous publication containing low-energy data for mutual neutralization low-energy data for mutual neutralization. Cross sections for other partial mutual neutralization processes calculated in Ref. [26] also deviate from the present data substantially. It is worth pointing out that the data of Ref. [26] not only missed the largest cross section at low energies, but also gave wrong relative relations between different partial cross sections. As discussed above, the reason is twofold: the electronic-structure calculations and the way to take into account multiple nonadiabatic regions. The second largest mutual neutralization cross section at low collision energies is one into the $\operatorname{Cs}(7 s)+\mathrm{H}$ final state. However, in the highenergy range, the cross section for mutual neutralization into

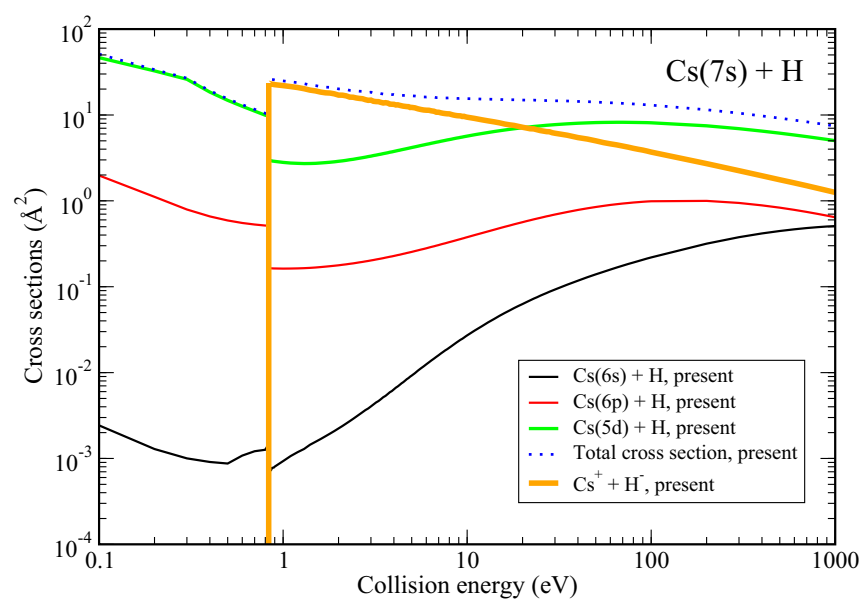

FIG. 5. (Color online) Electronic-excitation cross sections as a function of collision energy. For all transitions shown, the initial state is $\mathrm{Cs}(7 s)+\mathrm{H}$ and the final-state labels are given in the legend. Both neutral $\mathrm{Cs}(6 s, 6 p, 5 d)+\mathrm{H}$ and ionic $\mathrm{Cs}^{+}+\mathrm{H}^{-}$final states are considered. Total cross section refers to the sum over all final states considered in this figure. 


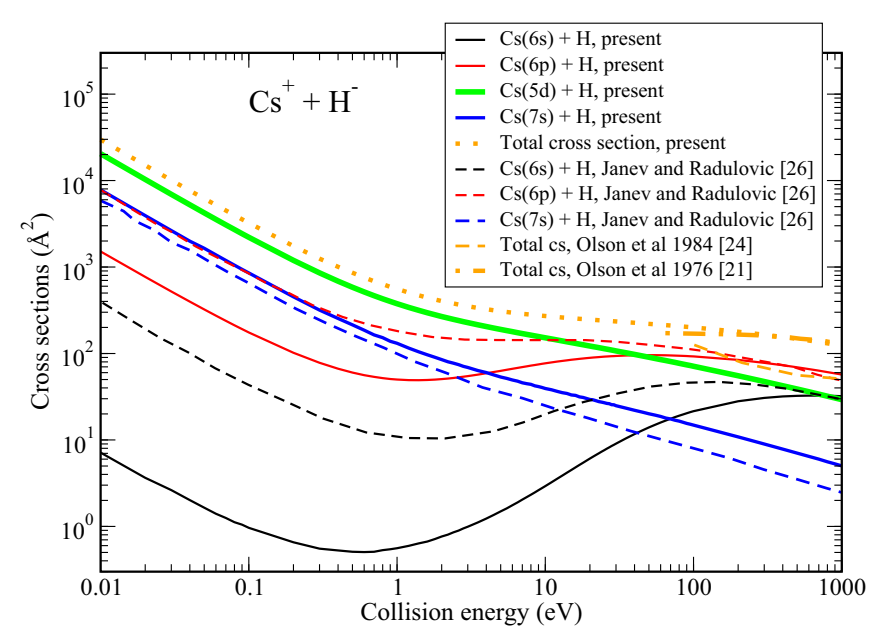

FIG. 6. (Color online) Mutual neutralization cross sections as a function of collision energy. For all transitions shown, the initial state is the ionic one $\mathrm{Cs}^{+}+\mathrm{H}^{-}$and the final-state labels are given in the legend. The neutral states $\mathrm{Cs}(6 s, 6 p, 5 d, 7 s)+\mathrm{H}$ are considered. Total cross section refers to the sum over all final states considered in this figure. The present results are compared with those of Olson et al. [21,24] and Janev and Radulović [26].

the $\mathrm{Cs}(6 p)+\mathrm{H}$ final state becomes the largest one exceeding the cross sections for the processes into both the $\mathrm{Cs}(5 d)+\mathrm{H}$ and the $\mathrm{Cs}(7 s)+\mathrm{H}$ final states.

The present total mutual neutralization cross sections can be compared with those from Refs. [21,24]. As discussed above, the calculations of Ref. [24] underestimated the cross sections because of less accurate electronic-structure calculations. This is clearly seen in Fig. 6. The results from Ref. [21] are in good agreement with the present total cross section.

The branching-probability-current method [34] allows one to calculate incoming and outgoing probability currents in each molecular state for every given collision, that is, for a given initial channel, a given collision energy and a given total angular momentum quantum number $J$. Calculated probability currents allows one to understand a reaction mechanism and specify regions that are mostly responsible for nonadiabatic transitions. The examples are presented in Figs. 7 and 8 for $\mathrm{Cs}(6 s)+\mathrm{H}$ and $\mathrm{Cs}^{+}+\mathrm{H}^{-}$collisions, respectively, for low collision energies, which correspond to the same total energy $E_{\text {total }}=3.5 \mathrm{eV}$ measured from the asymptotic ground-state potential and the same quantum number $J=0$. As discussed above, in the high-energy range, nonadiabatic transitions in $\mathrm{Cs}(6 s)+\mathrm{H}$ collisions are mainly determined by long-range $R \geqslant 10$ a.u. nonadiabatic regions, in particular, the nonadiabatic region around $R \approx 10$ a.u. between the ground $X^{1} \Sigma^{+}$and the first excited $A^{1} \Sigma^{+}$states. In the previous calculations, including Ref. [26] for low-energy collisions, practically only long-range nonadiabatic regions were taken into account. Figure 7 clearly shows that at low energies the nonadiabatic region around $R \approx 10$ a.u. does not change probability currents much, while several short-range nonadiabatic regions are responsible for redistribution of both incoming and outgoing currents between different molecular states. In particular, the regions at $R<3$ a.u. provide population of the final $\mathrm{Cs}(6 p)+\mathrm{H}$ state. Long-range regions are also involved

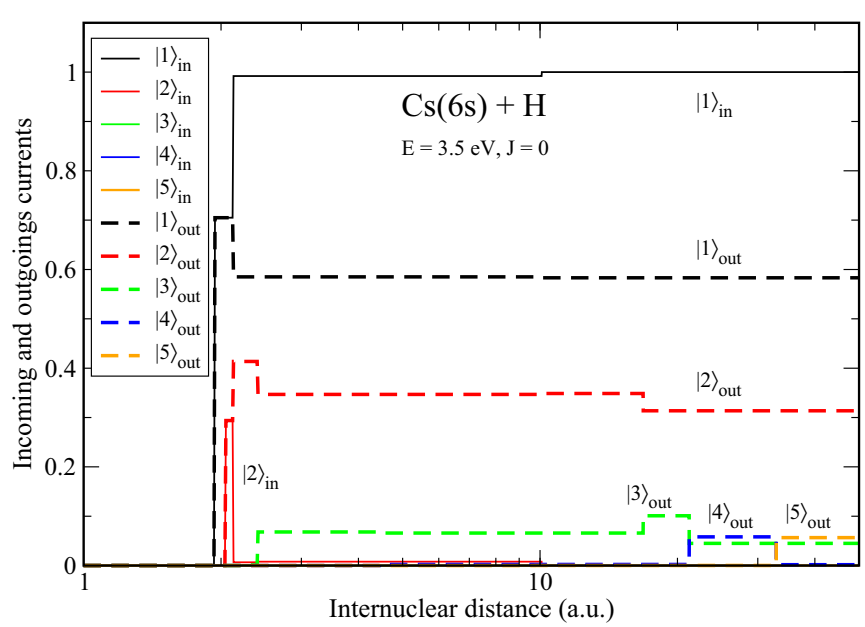

FIG. 7. (Color online) Incoming (thin solid lines) and outgoing (thick dashed lines) probability currents for the collision energy $E=$ $3.5 \mathrm{eV}$ and the total angular momentum quantum number $J=0$ for the initial state $\mathrm{Cs}(6 s)+\mathrm{H}$. The molecular-state labels are given in the legend.

in redistributions of outgoing currents, yielding populations of different final states. The situation is even more complicated in low-energy $\mathrm{Cs}^{+}+\mathrm{H}^{-}$collisions (see Fig. 8). Practically all long- and short-range nonadiabatic regions are responsible for redistribution of incoming and outgoing currents. In particular, several long- and short-range regions are involved in the population of the final $\mathrm{Cs}(5 d)+\mathrm{H}$ state, the state with the largest cross section in low-energy $\mathrm{Cs}^{+}+\mathrm{H}^{-}$collisions. Thus, the nonadiabatic nuclear dynamics at low energies is more complicated than that at high energies and this should be properly taken into account in scattering calculations. As mentioned above, this is one of the main sources for the deviation of the present results from the previous ones; another important source is the electronic-structure calculations.

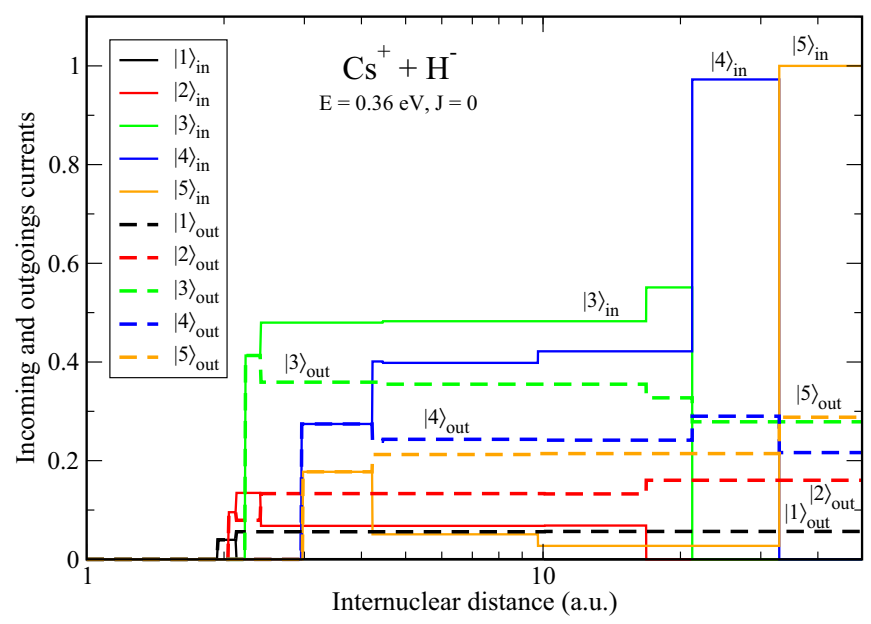

FIG. 8. (Color online) Incoming (thin solid lines) and outgoing (thick dashed lines) probability currents for the collision energy $E=$ $0.36 \mathrm{eV}$ and the total angular momentum quantum number $J=0$ for the initial state $\mathrm{Cs}^{+}+\mathrm{H}^{-}$. This energy corresponds to the same total energy as for the collision energy $3.5 \mathrm{eV}$ with the $\mathrm{Cs}(6 s)+\mathrm{H}$ initial channel. The molecular-state labels are given in the legend. 


\section{CONCLUSION}

The accurate $a b$ initio adiabatic potential energies [27] and the branching-probability-current method [34] for the nonadiabatic nuclear dynamics have been used to calculate inelastic cross sections for the excitation, deexcitation, ion-pair formation, and mutual neutralization processes in cesiumhydrogen collisions $\mathrm{Cs}(6 s, 6 p, 5 d, 7 s)+\mathrm{H}$ and $\mathrm{Cs}^{+}+\mathrm{H}^{-}$. Scattering calculations have been performed in the low-energy range from $0.01 \mathrm{eV}$ (or corresponding energy thresholds) to $1 \mathrm{keV}$.

It was shown that among the endothermic processes, the highest values of the partial cross sections correspond to the ion-pair formation processes when the collision energy is enough for populating the ionic molecular channel. The typical maximal ion-pair formation partial cross sections are of the order of $10 \AA^{2}$, although for $\operatorname{Cs}(7 s)+\mathrm{H}$ collisions the maximum reaches the value of $23 \AA^{2}$.

Among the exothermic processes in the low-energy range, the largest partial cross section corresponds to the mutual neutralization process into the $\mathrm{Cs}(5 d)+\mathrm{H}$ final state. The second largest low-energy cross section is for mutual neutralization into the $\mathrm{Cs}(7 s)+\mathrm{H}$ state. In the high-energy range, the cross section for mutual neutralization into the $\mathrm{Cs}(6 p)+\mathrm{H}$ state has the highest value. Of special interest is that the mutual neutralization cross sections are quite large, a fact that may be important for plasma modeling.

The calculated data are reliable and can be used for plasma modeling in different fields, including fusion plasma. In particular, the present data could be readily used by plasma physicists to estimate the effect of cesium seeding on hydrogen-negative-ion volume production in neutral beam injectors.

\section{ACKNOWLEDGMENTS}

We thank Dr. Marthe Bacal for continuous stimulation and support to this work. A.K.B. gratefully acknowledges partial support from the Russian Foundation for Basic Research (Grant No. 13-03-00163-a), the Russian Ministry of Education and Science and Université de Toulouse, UPS, for an invited professor grant.
[1] M. Bacal, A. Hatayama, and J. Peters, IEEE Trans. Plasma Sci. 33, 1845 (2005).

[2] M. Bacal, Nucl. Fusion 46, S250 (2006).

[3] M. Bacal, Rev. Sci. Instrum. 79, 02A516 (2008).

[4] M. Bacal, Chem. Phys. 398, 3 (2012).

[5] R. I. Hall, I. Čadež, M. Landau, F. Pichou, and C. Schermann, Phys. Rev. Lett. 60, 337 (1988).

[6] S. Béchu, D. Lemoine, M. Bacal, A. Bès, and J. Pelletier, in Proceedings of the First International Symposium on Negative Ions, Beams and Sources, edited by E. Surrey and A. Simonin, AIP Conf. Proc. No. 1097 (AIP, New York, 2009), p. 74.

[7] M. Bacal, R. McAdams, and B. Lepetit, in Proceedings of the Second International Symposium on Negative Ions, Beams and Sources, edited by Y. Takeiri and K. Tsumori, AIP Conf. Proc. No. 1390 (AIP, New York, 2011), p. 13.

[8] R. McAdam and E. Surrey, in Proceedings of the First International Symposium on Negative Ions, Beams and Sources (Ref. [6]), p. 89.

[9] P. Franzen, H. D. Falter, U. Fantz, W. Kraus, M. Berger, S. Christ-Koch, M. Fröschle, R. Gutser, B. Heinemann, S. Hilbert, S. Leyer, C. Martens, P. McNeely, R. Riedl, E. Speth, and D. Wünderlich, Nucl. Fusion 47, 264 (2007).

[10] P. G. Steen and W. G. Graham, Appl. Phys. Lett. 75, 2737 (1999).

[11] U. Fantz, H. D. Falter, P. Franzen, M. Bandyopadhyay, B. Heinemann, W. Kraus, P. McNeely, R. Riedl, E. Speth, A. Tanga, and R. Wilhelm, Fusion Eng. Design 74, 299 (2005).

[12] J. R. Hiskes and A. M. Karo, J. Appl. Phys. 67, 6621 (1990).

[13] J. R. Peterson, in Proceedings of the Fourth International Symposium on Production and Neutralizations of Negative Ions and Beams, edited by J. G. Alessi, AIP Conf. Proc. No. 158 (AIP, New York, 1987), p. 113.

[14] B. Lepetit, J. M. Launay, M. L. Dourneuf, and F. X. Gadéa, Chem. Phys. 117, 17 (1987).
[15] M. Nishiura, Y. Matsumoto, K. Tsumori, M. Wada, and T. Inoue, in Proceedings of the Second International Symposium on Negative Ions, Beams and Sources (Ref. [7]), p. 175.

[16] M. Bacal, F. E. Balghiti-Sube, L. I. Elizarov, and A. Y. Tontegode, Rev. Sci. Instrum. 69, 932 (1998).

[17] U. Fantz, H. D. Falter, P. Franzen, E. Speth, R. Hemsworth, D. Boilson, and A. Krylov, Rev. Sci. Instrum. 77, 03A516 (2006).

[18] T. J. Morgan, R. E. Olson, A. S. Schlachter, and J. W. Gallagher, J. Phys. Chem. Ref. Data 14, 971 (1985).

[19] F. W. Meyer, J. Phys. B 13, 3823 (1980).

[20] K. Miethe, T. Dreiseidler, and E. Salzborn, J. Phys. B 15, 3069 (1982).

[21] R. E. Olson, E. J. Shipsey, and J. C. Browne, Phys. Rev. A 13, 180 (1976).

[22] J. R. Hiskes, A. M. Karo, and P. A. Willmann, Phys. Lett. 68A, 221 (1978).

[23] R. E. Olson, Phys. Lett. 77A, 143 (1980).

[24] R. E. Olson, M. Kimura, and H. Sato, Phys. Rev. A 30, 1692 (1984).

[25] M. Kimura, Appl. Surf. Sci. 253, 6641 (2007).

[26] R. K. Janev and Z. M. Radulović, Phys. Rev. A 17, 889 (1978).

[27] W. Zrafi, B. Oujia, and F. X. Gadéa, J. Phys. B 39, 3815 (2006).

[28] G. H. Jeung, F. Spiegelmann, J. P. Daudey, and J. P. Malrieu, J. Phys. B 16, 2659 (1983).

[29] M. Carneil, S. D. Peyerimhoff, and B. A. Heß, Z. Phys. D 13, 317 (1989).

[30] H. Croft, A. S. Dickinson, and F. X. Gadéa, J. Phys. B 32, 81 (1999).

[31] H. Croft, A. S. Dickinson, and F. X. Gadéa, Mon. Not. R. Astron. Soc. 304, 327 (1999).

[32] A. K. Belyaev, P. S. Barklem, A. S. Dickinson, and F. X. Gadéa, Phys. Rev. A 81, 032706 (2010). 
[33] A. K. Belyaev, P. S. Barklem, A. Spielfiedel, M. Guitou, N. Feautrier, D. S. Rodionov, and D. V. Vlasov, Phys. Rev. A 85, 032704 (2012).

[34] A. K. Belyaev, Phys. Rev. A 88, 052704 (2013).

[35] A. K. Belyaev and O. V. Lebedev, Phys. Rev. A 84, 014701 (2011).

[36] H. M. Hedberg, S. Nkambule, and Å. Larson, J. Phys. B 47, 225206 (2014).

[37] M. Stenrup, Å. Larson, and N. Elander, Phys. Rev. A 79, 012713 (2009).
[38] A. K. Belyaev, Astron. Astrophys. 560, A60 (2013).

[39] A. K. Belyaev, L. Augustovičová, P. Soldán, and W. P. Kraemer, Astron. Astrophys. 565, A106 (2014).

[40] A. K. Belyaev and A. Z. Devdariani, Opt. Spektrosk. 45, 448 (1978) [Opt. Spectrosc. 45, 253 (1978)].

[41] A. K. Belyaev, A. Z. Devdariani, V. A. Kostenko, and Y. A. Tolmachev, Opt. Spektrosk. 49, 633 (1980) [Opt. Spectrosc. 49, 345 (1980)].

[42] M. Y. Ovchinnikova, Sov. Phys. JETP 37, 68 (1973). 